\title{
Detection of "Hidden" Antimicrobial Drug Resistance
}

Qingfeng $\mathrm{Hu}^{2 \mathrm{a}}$, Yan $\mathrm{Yu}^{1}{ }^{1}$, Dongshi $\mathrm{Gu}^{1 \mathrm{a}}$, $\mathrm{Li} \mathrm{Xie}^{3}$, Xingrou $\mathrm{Chen}^{1}$, Ning $\mathrm{Xu}^{1}$, Jennifer Jin Ruan ${ }^{4}$, Christopher Dowson ${ }^{5}$, Benfang Helen Ruan ${ }^{1 *}$

${ }^{1}$ College of Pharmaceutical Science, Collaborative Innovation Center of Yangtza River Delta Region Green Pharmaceuticals, IDD \& CB, Zhejiang University of Technology, 18 Chaowang Road, Xiachengqu, Hangzhou, Zhejiang, 310014, China

${ }^{2}$ Clinical Diagnostic lab, Renming Hospital of Zhejiang Province, Hangzhou, 310014, China

${ }^{3}$ Center for M. tuberculosis Research, Hangzhou, 310019 China

${ }^{4}$ Department of Surgery, Memorial Sloan Kettering Cancer Center, NYC, 10009, USA

${ }^{5}$ School of Life Sciences, University of Warwick. CV47AJ, U. K.

\section{*Corresponding author}

\section{Benfang Helen Ruan (Ph. D)}

Professor of Pharmaceutical Science

College of Pharmaceutical Science, Collaborative Innovation Center of Yangtza River Delta Region, IDD \& CB, Green Pharmaceuticals, Zhejiang University of Technology

E-mail: ruanbf@zjut.edu.cn; ruanbf@yahoo.com

Tel: 86-18357023608

Fax: (0086) 571-88871098

aCurrent address: 18 Chaowang Road, Xiachengqu, Hangzhou, Zhejiang, China, 310014 


\section{Supplementary figures}

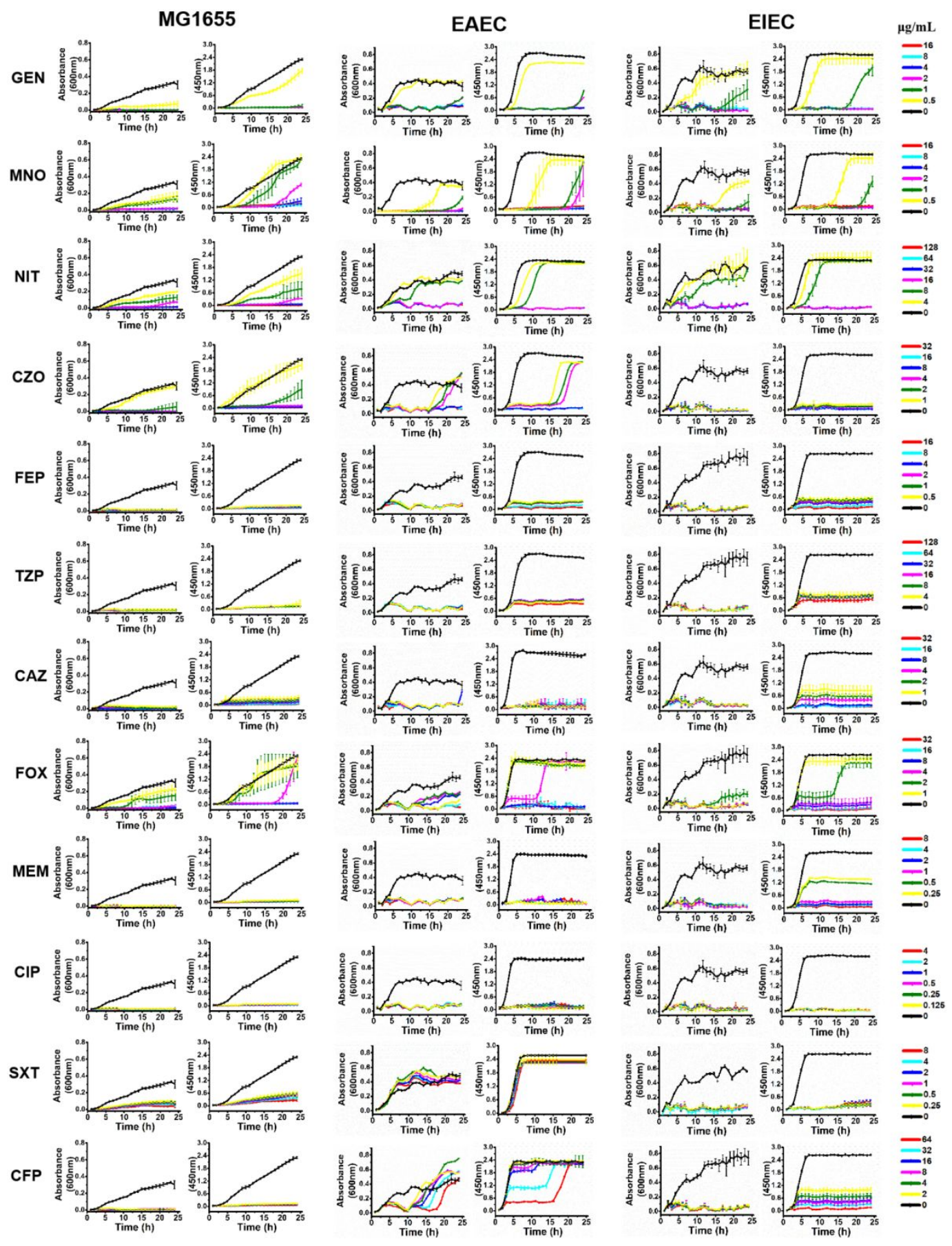

Figure S1. DIP curves collected by the turbidity $\left(\mathrm{OD}_{600 \mathrm{~nm}}\right)$ and the EZMTT $\left(\mathrm{OD}_{450 \mathrm{~nm}}\right)$ methods, showing the growth inhibitions of the wild type $E$. coli (MG1655) and the clinically isolated $E$. coli strains (EAEC, EIEC) by 12 antibiotic medicine. Results are representative of at least 3 experiments in duplicates. 

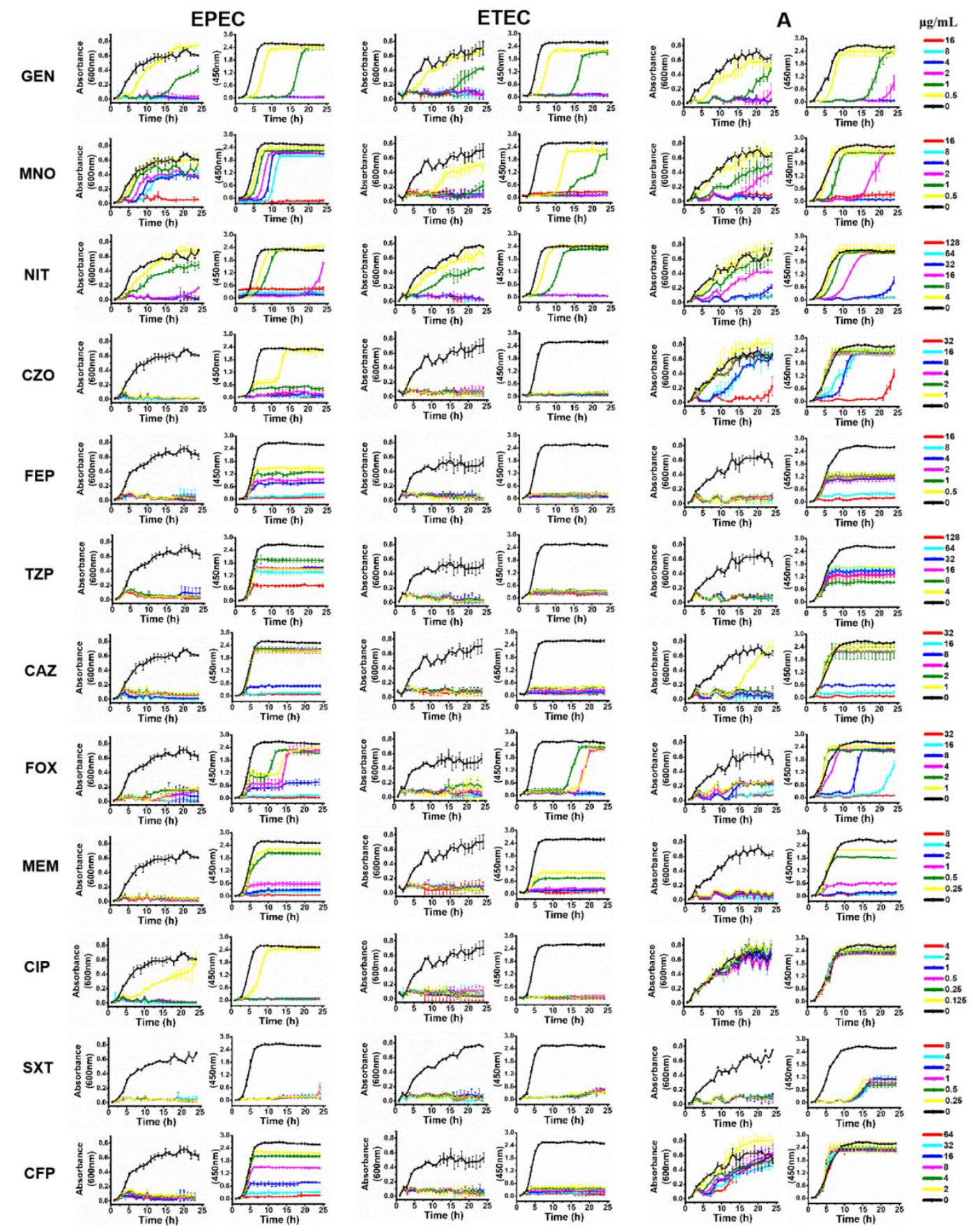

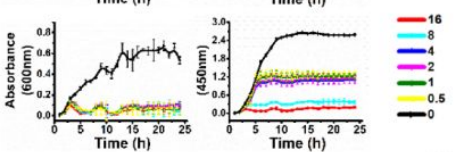
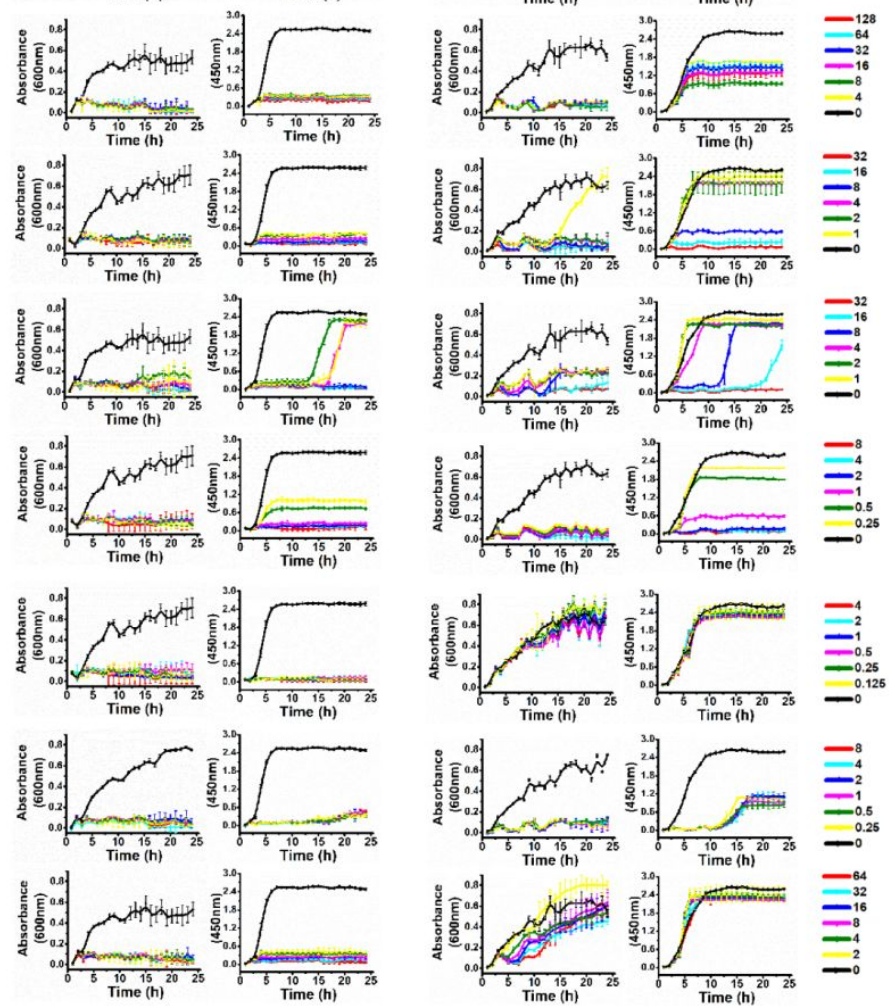

Figure S2. DIP curves collected by the turbidity $\left(\mathrm{OD}_{600 \mathrm{~nm}}\right)$ and the EZMTT $\left(\mathrm{OD}_{450 \mathrm{~nm}}\right)$ methods, showing the growth inhibitions of the clinically isolated E. coli strains (EPEC, ETEC, A) by 12 antibiotic medicine. Results are representative of at least 3 experiments in duplicates. 